\title{
LIV. Diffusion cells in ionized gases
}

\section{E.B. Wood M.A. , O.A. de Long B.S. \& K.T. Compton Ph.D.}

To cite this article: E.B. Wood M.A. , O.A. de Long B.S. \& K.T. Compton Ph.D. (1916) LIV. Diffusion cells in ionized gases, Philosophical Magazine Series 6, 32:191, 499-504, DOI: 10.1080/14786441608635596

To link to this article: http://dx.doi.org/10.1080/14786441608635596

$$
\text { 曲 Published online: } 08 \text { Apr } 2009 .
$$

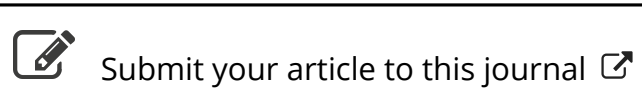

\section{Џ Article views: 2}

Q View related articles $\widetilde{ }$

4 Citing articles: 1 View citing articles 준 
used. With gypsum this constant was found to be $7 \cdot 621 \cdot 10^{-8}$ $\mathrm{cm}$., being the mean of two determinations only differing by 0.16 per cent.

The values of the wave-lengths are put together in Table I., and in Plate XI. a graphical representation of the results (from zirconium to uranium) after the relation of Moseley is given. As seen, 14 line-groups seem to be sure in the L-series. Of these the l-group was discovered by Siegbahn *, who has examined these lines in the elements tungsten-uranium. The graphical representation shows further, that only in some cases $\left(\alpha_{2}, \alpha_{1}, \beta_{2}\right)$ a Moseley relation holds good for the whole range, while the other groups generally form curves slightly convex to the $\mathrm{X}$-axis. The order of the rare earths here found agrees with that generally adopted. The order Te-I found by Siegbahn + for the $\mathrm{K}$-series is also verified here.

I have much pleasure in recording my thanks to Dr. M. Siegbahn for suggesting this work to me.

Physical Laboratory, Unirersity of Lund, June 1916.

LIV. Diffusion Cells in Ionized Gases. By E. B. Wood, M.A., O. A. DE Long, B.S., and K. T. Compton, Ph.D., Assistant Professor of Physics, Princeton University $\ddagger$.

$\mathrm{N}$ a recent paper $\S \mathrm{Mr}$. W. H. Jenkinson has shown 1 very conclusively that an electromotive force is set up between two similar electrodes in a gas if the degree of ionization of the gas surrounding the two electrodes is unequal. The effect of this phenomenon on the relative potentials of an electrode and the survounding gas had already been investigated by Professor Juhn Zeleny\|. The interpretation given by $\mathrm{Mr}$. Jenkinson to his results, however, appears to us deserving of further examination.

Following the analogy of Nernst's treatment of electrolytic cells by the concept of solution tension and solution

* Verh. d. Phys. Ges. xviii. p. 150 (1916).

† Verh. d. Phys. Ges. xviii. p. 39 (1916).

I Communicated by the Authors.

\$ "Concentration Cells in Ionized Gases," Phil. Mag. xxriii. p. 685 (1914).

II Phil. Mag. xlvi. p. 134 (1898). 
pressure, M:. Jenkinson interpreted his results by the equation

$$
\mathrm{E}=\frac{\mathrm{RT}}{e}(2 x-1) \log \frac{p_{1}}{p_{2}}, \ldots . . .
$$

where $p_{1}$ and $p_{2}$ are the partial pressures of the ions of either kind just outside the electrodes, $x$ is the proportion of the total current carried by the positive ions, and $\mathrm{E}$ is the electromotive force resulting from the unequal ionic concentrations at the two electrodes. According to this equation the sign of the electromotive force should depend on the value of $x$, $i . e$. on the kind of ion, positive or negative, which effects the greater transfer of electricity.

From the sign of the electromotive force observed in his experiments (the electrode exposed to the ionizing radiations always acquiring a positive charge), Mr. Jenkinson concluded that it must be the positive ions which determine the potential step between a metal and the surrounding gas. He takes from his experiments support of the view that a layer of occluded gas at the surface of a metal is essential to the production of a contact potential effect, and that positive ions only pass from this layer to the surrounding gas.

In view of the increasing importance of discovering the conditions which exist at the surface of a metal, we feel justitied in pointing out a much more probable interpretation of Mr. Jenkinson's experiments, which involves neither the formation of an occluded gas layer nor the passage of electricity to and from a metal by the sole agency of positive ions.

Theory.-Consider the simplest case, that of two infinite parallel metal plates and a source of ionizing radiations which ionizes the intervening gas more strongly near one plate than the other. If the two plates are originally at tle same potential, the ions will move by diffusion from the regions of greater to those of less concentration; but this process will never produce uniform ionic concentration in all parts of the gas because of recombinations which take place simultaneously with diffusion. In this respect the present case differs from the similar case of an electrolyte.

If the positive and negative ions diffuse at the same rate the plates will obviously remain at equal potentials; but if the negative ions diffuse at a more rapid rate than the positive ions, as is usually the case, the plate in the region of less ionic concentration will acquire a negative charge and a negative potential with respect to the other plate. If the plates are insulated, this process will continue until the 
electric field is just sufficient to cause the positive and negative ions to drift at equal average rates toward the negative plate, numbers of them recombining as they drift.

Such an explanation of the electromotive force of concentration-cells in ionized gases accounts for the sign of the electromotive force observed by $\mathrm{Mr}$. Jenkinson without introducing any hypothesis regarding the phenomena at the surface of the metal. That it is the correct explanation is indicated by the foilowing experiments.

The process just described may be put in a form for quantitative experimental test by applying Prof. J. J. Thomson's well-known equations for the time rate of increase of the number $n$ of ions per unit volume at any point in the gas :

$$
\begin{aligned}
& \frac{d n_{1}}{d t}=q-\alpha n_{1} n_{2}+\mathrm{D}_{1} \frac{d^{2} n_{1}}{d x^{2}}-u_{1} \frac{d}{d x}\left(\mathrm{X} n_{1}\right), \\
& \frac{d n_{2}}{d t}=q-\alpha n_{1} n_{2}+\mathrm{D}_{2} \frac{d^{2} n_{2}}{d x^{2}}+u_{2} \frac{d}{d x}\left(\mathrm{X} n_{2}\right) ;
\end{aligned}
$$

where subscripts 1 and 2 refer to the positive and negative ions, respectively. $q$ represents the rate of production of ions by the ionizing ayent; $\alpha$ and $D$ are the coefficients of recombination and diffusion, respectively; $u$ is the ionic velocity in a field of unit intensity; $\mathrm{X}$ is the electric intensity, and $x$ is the coordinate normal to the plates.

We may eliminate the terms involving $q$ and $\alpha$ by subtraction and integrate the resulting equation. The integration constant vanishes, since the current through the gas vanishes in the final steady state. By Poisson's equation it follows that in the steady state $n_{1}=n_{2}$ at any point in the gas. Making this substitution and integrating again, we find

$$
\mathrm{X} d=\frac{\mathrm{D}_{1}-\mathrm{D}_{2}}{u_{1}+u_{2}} \log \frac{n_{a}}{n_{0}},
$$

where $d$ is the distance between the plates and $n_{0}$ and $n_{\alpha}$ are the ionic concentrations in the immediate vicinities of the plates. $\mathrm{X} d=\mathrm{E}$, where $\mathrm{E}$ is the electromotive force, and $\mathrm{D}=\mathrm{RT} u / e^{*}$. Thus we have

$$
\mathrm{E}=\frac{\mathrm{RT}}{e} \frac{u_{1}-u_{2}}{u_{1}+u_{2}} \log \frac{n_{\alpha}}{n_{0}} . . . . .
$$

This equation is given in Winkelmann's Handluch der Physik, 2 ed. vol. iv. p. 616 , being there derived by a consideration of partial pressures as in the case of an electrolyte.

* J. J. Thomson, 'Conduction of Electricity through Gases,' p. 43. 
When a current flows through the gas

$$
\frac{u_{1}}{u_{1}+u_{2}} \text { and } \frac{u_{2}}{u_{1}+u_{2}}
$$

give the proportions of the electricity carried by the positive and negative ions, respectively. Furthermore, the partial ionic pressures $p$ are proportional to the ionic concentrations $n$. Thus we may write equation (2) in the form of equation (1) used by Mr. Jenkinson. The interpretation of $x$ here suggested, however, is typical of a diffusion-cell rather than an ordinary concentration-cell such as Mr. Jenkinson considered.

Experimental Tests.-We tested the validity of equation (2) by an apparatus whose essential features are shown in the diagram. Brass plates $A$ and $B$ were surrounded by guardrings $G$, mounted on ebonite blocks and enclosed in the
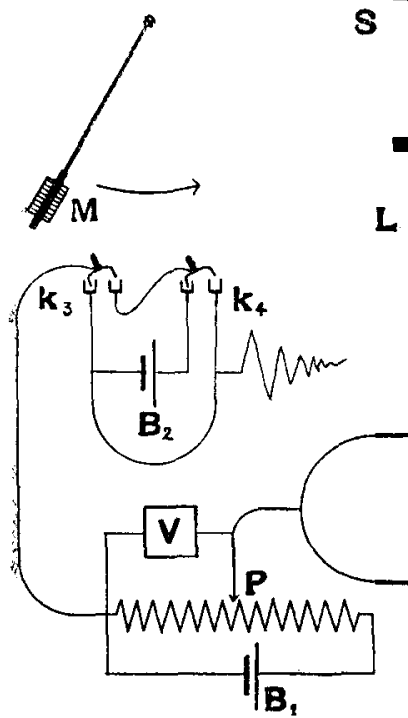

thick-walled lead box L L, which was lined with brass to avoid disturbances due to contact difference of potential. A tube of radium at $R$ was also enclosed in a lead box provided with a fan-shaped opening. This box could be set against stops $S$ so as to expose the gas near either plate to the ionizing $\gamma$ rays. The potential divider $P$ enabled the potential of the plate $A$ to be adjusted until the electrometer $\mathbf{E}$ connected with the plate $B$ showed no deflexion. The difference 
between the voltmeter readings with the radium in the two positions gave twice the electromotive force of the diffusioncell. An insulated switch, not shown in the figure, enabled the connexions to the two sets of plates to be interchanged.

By means of two switches $k_{3}$ and $k_{4}$, actuated by an electromagnet pendulum-bob $\mathrm{M}$, a field of two volts from the battery $B_{2}$ could be suddenly applied to the plate $A$ for a definite small interval of time, usually about 0.05 second. During this interval ions of one sign within a fraction of a millimetre of the plate $B$ were swept into the plate, producing a deflexion of the electrometer. This could be repeated with the ionizing source acting near the other plate, or with the connexions to $A$ and $B$ reversed. The ratio of these deflexions gave the ratio $n_{a} / n_{0}$. All other quantities in equation (2) being known, these measurements enabled a quantitative test of the equation to be made.

Unfortunately our tube of radium did not produce sufficiently strong jonization to permit an accurate measure of the ratio $n_{\alpha} / n_{0}$, the electrometer deflexions being too small in comparison with a somewhat irregular shift due to an unbalanced induction effect while the field was changed by the switches $k_{3}$ and $k_{4}$. We were consequently forced to use an $\mathrm{X}$-ray tube as the ionizing source in this part of the experiment, and the measurements were subject to the somewhat erratic variations in its action. However, with this arrangement, we obtained results of which the following are examples. $n_{0}$ and $n_{a}$ are in terms of the electrometer deflexions noted just after the fall of the pendulum $M$. The

TABLE I.

\begin{tabular}{|l|l|l|l|}
\hline$n_{0}$. & $n_{\alpha .}$ & $\mathrm{E}$ (calc.). & $\mathbf{E}$ (obs.). \\
\hline 24 & 7 & 0.00497 & 0.0042 \\
18.5 & 5 & 000528 & $\theta \cdot 0034$ \\
24 & 5 & 0.00633 & 0.0067 \\
\hline
\end{tabular}

electrometer sensitiveness, about 5000 divisions per volt, was such as to make the apparatus very sensitive to disturbing influences, so that we feel that our measurements may be taken as a fair support of the theory. Doubtless, by taking greater precautions to insure steady strong ionization and by increasing the distance between the plates $A$ and $B$ so as to increase the amount of recombination, and therefore the ratio $n_{a} / n_{0}$, the experimental test could have been made more accurate. 
We tested equation (2) in another manner, by substituting dry carbon dioxide gas for air between the plates. In this case, the difference between the ionic velocities $u_{1}$ and $u_{2}$ is much less than in air, from which we should expect a smaller electromotive force. In order to avoid large changes in the strength of the ionizing source during the substitution of one gas for the other, we used the radium for the ionizing source in this test and increased the electrometer sensitiveness to about 15,000 divisions per volt. The averages of a number of consistent measurements gave $\mathrm{E}=0.00090$ volt for air and $\mathrm{E}=0.00035$ volt for carbon dioxide. This difference is in the right direction and of the general size predicted by equation (2), though an exact prediction cannot be made because of the differenee in the degrees of ionization and recombination in the two gases.

Finally we tried the effect of filling the apparatus with moist carbon dioxide, in which case the sign of the electromotive force should be reversed, owing to the fact that the positive ions diffuse more rapidly than the negative ions in this gas. This test, however, was unsuccessful because the insulation broke down before the measurements could be made.

In conclusion we might mention an attempt to test equation (2) without the necessity for measuring the ionic concentrations at the plates by the aid of the equation

$$
\frac{d^{2} n}{d x^{2}}+\mathrm{A} \frac{d n}{d x}+\mathrm{B} n^{2}=0
$$

which follows from Thomson's equations given earlier in the paper if we put $A=u_{2} X / D_{2}$ and $B=-\alpha / D_{2}$. This equation is of the type of the equation of motion of a particle moving, subject to friction, under the action of a force proportional to the square of the distance from a fixed point, and has not been solved. Approximate solutions indicated that tho ratio $n_{a} / n_{0}$ is not independent of $n_{0}$, as we might have anticipated. Thus even with an exact solution of this differential equation it would still be necessary to make some sort of measurement of the ionic concentration, so that this method was not pursued further.

We are glad to acknowledge our indebtedness to Professor E. P. Adams for suggesting approximate solutions of the differential equation mentioned above, and to Professor H. L. Cooke for suggesting the method which we employed for measuring the relative values of the ionic concentrations at the plates.

Palmer Physical Laboratory,

Princeton, N. J., U.S.A. 